PROCEEDINGS OF THE

AMERICAN MATHEMATICAL SOCIETY

Volume 121, Number 4, August 1994

\title{
THE UNITABILITY OF $l$-PRIME LATTICE-ORDERED RINGS WITH SQUARES POSITIVE
}

\author{
MA JINGJING
}

(Communicated by Lance W. Small)

\begin{abstract}
It is shown that an $l$-prime lattice-ordered ring with squares positive and an $f$-superunit can be embedded in a unital $l$-prime lattice-ordered ring with squares positive.
\end{abstract}

In [1, p. 325] Steinberg asked if an $l$-prime $l$-ring with squares positive and an $f$-superunit can be embedded in a unital $l$-prime $l$-ring with squares positive. In this paper we show that the answer is yes.

If $R$ is a lattice-ordered ring ( $l$-ring) and $a \in R^{+}$, then $a$ is called an $f$ element of $R$ if $b \wedge c=0$ implies $a b \wedge c=b a \wedge c=0$. Let $T=T(R)=$ $\{a \in R:|a|$ is an $f$-element of $R\}$. Then $T$ is a convex $f$-subring of $R$, and $R$ is a subdirect product of totally ordered $T-T$ bimodules [2, Lemma 1]. $R$ is an $f$-ring precisely when $T=R$. Throughout this paper $T$ will denote the subring of $f$-elements of $R$. An element $e \geq 0$ of an $l$-ring $R$ is called a superunit if $e x \geq x$ and $x e \geq x$ for each $x \in R^{+} ; e$ is an $f$-superunit if it is a superunit and an $f$-element. $R$ is infinitesimal if $x^{2} \leq x$ for each $x$ in $R^{+}$. The $l$-ring $R$ is called $l$-prime if the product of two nonzero $l$-ideals is nonzero and $l$-semiprime if it has no nonzero nilpotent $l$-ideals. An $l$-ring $R$ is called squares positive if $a^{2} \geq 0$ for each $a$ in $R$.

Let $A$ be any ring and $a \in A$. If $a$ satisfies $a b=b a=n b$ for some fixed integer $n$ and all $b \in A$, then $a$ is said to be an $n$-fier of $A$ and $n$ is said to have an $n$-fier $a$ in $A$. Let $K=\{n \in Z: n$ has $n$-fiers in $A\}$. Then $K$ is an ideal in the ring $Z$ of integers. The ideal $K$ is called the modal ideal of $A$; its nonnegative generator $k$ is called the mode of $A$. If $R$ is an $f$-ring with mode $k>0$, then $R$ has a unique $k$-fier $x \geq 0$ [3, III, Lemma 2.1].

Let $R$ be an $l$-ring, and let $S=S(R)=\left\{a \in R:|a| \geq d, \forall d \in T^{+}\right\}$ and $T^{\perp}=\left\{a \in R:|a| \wedge d=0, \forall d \in T^{+}\right\}$. It is clear that $T^{\perp}$ is a convex $l$-subgroup of $R$.

The referee has pointed out to us that the following lemma, on which our arguments are based, is a special case of [4, Lemma 6.2], and P. Conrad attributes it to A. H. Clifford.

Received by the editors July 20, 1992 and, in revised form, October 30, 1992.

1991 Mathematics Subject Classification. Primary 06F25; Secondary 16A86.

Key words and phrases. Lattice-ordered ring, $l$-prime $l$-ring, unitability, squares positive. 
Lemma 1. Let $R$ be an l-ring which contains nonzero f-elements and $T$ a totally ordered subring of $R$. Then $R=S \cup\left(T \oplus T^{\perp}\right)$, where the direct sum is regarded as the direct sum of l-subgroups and $S \cap\left(T \oplus T^{\perp}\right)=\varnothing$.

Proof. If $a \in R$ and $a \notin S$, then $|a| \wedge b<b$ for some $b \in T^{+}$. Let $a_{1}=|a|-|a| \wedge b$ and $b_{1}=b-|a| \wedge b$. Then $a_{1} \wedge b_{1}=0$, and hence $\left(a_{1} \wedge d\right) \wedge b_{1}=0$ for each $d \in T^{+}$. Since $\left(a_{1} \wedge d\right), b_{1} \in T$, which is a totally ordered ring, and $b_{1}>0$, we have $a_{1} \wedge d=0$ for each $d \in T^{+}$, and hence $a_{1} \in T^{\perp}$. Since $0 \leq a^{+}, a^{-} \leq|a|=a_{1}+(|a| \wedge b)$, there are $a_{2}, a_{3}, b_{2}, b_{3} \in R$ such that $0 \leq a_{2}, a_{3} \leq a_{1}, 0 \leq b_{2}, b_{3} \leq|a| \wedge b, a^{+}=a_{2}+b_{2}, a^{-}=a_{3}+b_{3}$, and $a=a^{+}-a^{-}=\left(b_{2}-b_{3}\right)+\left(a_{2}-a_{3}\right)$. But $T$ and $T^{\perp}$ are the convex $l$-subgroups of $R$, so $a \in T \oplus T^{\perp}$.

If $a \in S \cap\left(T \oplus T^{\perp}\right)$, then $a=a_{1}+a_{2}$, where $a_{1} \in T$ and $a_{2} \in T^{\perp}$, and hence $2\left|a_{1}\right| \leq|a| \leq\left|a_{1}\right|+\left|a_{2}\right|$ since $a \in S$. Thus $a_{1}=0$ and $a=a_{2}$, and hence $T=\{0\}$. This contradicts $T \neq\{0\}$, so $S \cap\left(T \oplus T^{\perp}\right)=\varnothing$.

Let $R$ be an $l$-ring as in Lemma 1 and $a \in R$. If $a \in T \oplus T^{\perp}$, then $a$ can be uniquely represented as the sum of elements of $T$ and $T^{\perp}$, and we may write $a=a_{T}+a_{T^{\perp}}$, where $a_{T} \in T$ and $a_{T^{\perp}} \in T^{\perp}$ are respectively called the components of $a$ in $T$ and in $T^{\perp}$. It is clear that $a \vee 0=\left(a_{T} \vee 0\right)+\left(a_{T^{\perp}} \vee 0\right)$.

Throughout this paper the following fact is used frequently. Let $R$ be an $l$-ring and $e$ an $f$-superunit of $R$. Then, for each $x \in R, x \geq 0$ if and only if $e x \geq 0$ or $x e \geq 0$. More generally, if $0<a \in T$ is a regular element (or a non-zero-divisor), then, for each $x \in R, x \geq 0$ if and only if $a x \geq 0$ or $x a \geq 0$.

Lemma 2. Let $R$ be an l-ring with an $f$-superunit and $T$ a totally ordered ring. Let $e$ be an $f$-superunit of $R$.

(a) If $0<b \in S$, then $n e+b e \geq 0$ for each $n \in Z$.

(b) If $b \in S$ and $m e+b e \geq 0$ for some $m \in Z$, then $b>0$.

Proof. (a) Since $0<b \in S$, we have $-n e \leq|n| e \leq|n| e^{2} \leq b e$ for each $n \in Z$.

(b) Let $m e+b e \geq 0$ for some $m \in Z$. If $m \leq 0$, then $b e \geq-m e \geq 0$, so $b e=|b e|=|b| e$; that is, $(|b|-b) e=0$. But $e$ is an $f$-superunit, so $b=|b|>$ 0 . If $m>0$, then $m e \geq-b e$, and hence $m e \geq(-b e) \vee 0=(-b \vee 0) e \geq-b \vee 0$, so $-b \vee 0 \in T$. Since $b \vee 0=b+(-b \vee 0)$ and $b \in S$, we have $b \vee 0 \in S$ by Lemma 1. Thus $b+(-b \vee 0)=b \vee 0>-b \vee 0$; that is, $b>0$.

Lemma 3. Let $R$ be an l-ring with an $f$-superunit and $T$ a totally ordered ring. If the mode of $R$ is $n$ and the mode of $T$ is $k$, then $n=k$ and the $k$-fier of $R$ equals the $k$-fier of $T$.

Proof. Let $e$ be an $f$-superunit of $R$. If $x$ is a $k$-fiber of $T$, then $k d=$ $d x=x d$ for each $d \in T$, especially $k e=e x=x e$. Thus $e(k a)=e x a$ and $(k a) e=a x e$ for each $a \in R$. Since $e$ is an $f$-superunit of $R, k a=x a=a x$ for each $a \in R$, so $n \mid k$. Let $y$ be an $n$-fier of $R$. Then $n a=a y=y a$ for each $a \in R$. By Lemma 1 we have $R=S \cup\left(T \oplus T^{\perp}\right)$. If $y \in S$, then since $e$ is an $f$-superunit of $R$ and $n e=e y=y e$, we have $n e=|n e|=|e y|=e|y| \geq$ $|y| \geq(n+1) e$. This is a contradiction, so $y \in T \oplus T^{\perp}$. Let $y=y_{T}+y_{T^{\perp}}$. Then $n e=e y=e y_{T}+e y_{T^{\perp}}$, and hence $n e-e y_{T}=e y_{T^{\perp}} \in T \cap T^{\perp}=\{0\}$, so $y_{T^{\perp}}=0$ and $y=y_{T} \in T$. Thus $k \mid n$, and we have $k=n$. 
Lemma 4. Let $R$ be an l-ring which has an $f$-superunit and $T$ a totally ordered ring. If $e$ is an $f$-superunit of $R$ and $n \in Z, b \in T \oplus T^{\perp}$ satisfy ne $+b e \geq 0$, then $b_{T^{\perp}} \geq 0$ and $n e+b_{T} e \geq 0$.

Proof. If $n e+b e \geq 0$, then $\left(n e+b_{T} e\right)+b_{T^{\perp}} e \geq 0$. Thus $n e+b_{T} e \geq 0$ and $b_{T^{\perp}} e \geq 0$, so $b_{T^{\perp}} \geq 0$.

Lemma 5. Let $R$ be an l-ring with squares positive and $a \in T^{\perp}$ or $S$. Then $d|a| \leq a^{2}$ and $|a| d \leq a^{2}$ for each $d \in T^{\perp}$. If $R$ has an $f$-superunit, then $n|a| \leq a^{2}$ for each $n \in Z^{+}$.

Proof. Since $R$ is squares positive, $0 \leq(a \pm b)^{2}$ yields $|a d+d a| \leq a^{2}+d^{2}$. But $R$ is a $T$ - $T f$-bimodule, and $|a d|+|d a|=|a d+d a|$ holds in any totally ordered $T$-T bimodule which is a homomorphic image of $R$ since $0 \leq d \in T$; so it also holds in $R$ [2, Lemma 4]. If $a \in T^{\perp}$, then $|a| d=|a d| \leq|a d|+|d a|=$ $|a d+d a| \leq a^{2}+d^{2}$ and $|a| \wedge d=0$ imply $|a| d=|a| d \wedge\left(a^{2}+d^{2}\right) \leq\left(|a| d \wedge a^{2}\right)+$ $\left(|a| d \wedge d^{2}\right)=|a| d \wedge a^{2} \leq a^{2}$. Similarly, $d|a| \leq a^{2}$. If $a \in S$, then $d \leq|a|$ for each $d \in T^{+}$, and hence $|a| d+d|a|=|a d|+|d a|=|a d+d a| \leq a^{2}+d^{2} \leq$ $a^{2}+d|a|$. Thus $|a| d \leq a^{2}$. Similarly, $d|a| \leq a^{2}$. If $e$ is an $f$-superunit of $R$, then, as in the previous paragraph, $n|a| \leq n e|a| \leq a^{2}$ for each $n \in Z^{+}$.

Theorem 1. An l-prime l-ring $R$ with squares positive and an $f$-superunit can be embedded in a unit l-prime l-ring with squares positive.

Proof. We first note that $R$ is a domain [2, Theorem 1], and hence $T$ is a totally ordered ring. Thus $R=S \cup\left(T \oplus T^{\perp}\right)$ by Lemma 1. Let $e$ be an $f$-superunit of $R$.

Let $\bar{R}$ be the ring obtained by freely adjoining the integers to $R$, and let $k$ be the mode of $T$ and $x$ the unique $k$-fier of $T$. Then $k$ is the mode of $R$ and $x$ the unique $k$-fier of $R$ by Lemma 3. Let $I(k, x)$ be the set of all integral multiples of $(k,-x) \in \bar{R}$. Then $I(k, x)$ is an ideal of $\bar{R}$. Denote the ring $\bar{R} / I(k, x)$ by $R^{\prime}$ which has identity $(\overline{1,0})$. Since $R$ is a domain, it is well known that $R^{\prime}$ is a domain. Consider the subset $\left(R^{\prime}\right)^{+}$of $R^{\prime}$ defined by $(\overline{n, a}) \in\left(R^{\prime}\right)^{+}$if and only if $n c+a c \geq 0$ for all $c \in R^{+}$, which is equivalent to $n e+a e \geq 0$. Since $n c+a c \geq 0$ if and only if $(n+m k) c+(a-m x) c \geq 0$ for each $m \in Z,\left(R^{\prime}\right)^{+}$is well defined. It is obvious that $\left(R^{\prime}\right)^{+}$has the following properties:

(i) $(\overline{0,0}) \in\left(R^{\prime}\right)^{+}$,

(ii) $\left(R^{\prime}\right)^{+} \cap\left[-\left(R^{\prime}\right)^{+}\right]=\{0\}$,

(iii) $\left(R^{\prime}\right)^{+}+\left(R^{\prime}\right)^{+} \subseteq\left(R^{\prime}\right)^{+}$,

(iv) $\left(R^{\prime}\right)^{+}\left(R^{\prime}\right)^{+} \subseteq\left(R^{\prime}\right)^{+}$.

Hence, the ring $R^{\prime}$ is a partially ordered ring, and $a^{\prime} \leq b^{\prime}$ if and only if $\left(b^{\prime}-a^{\prime}\right) \in\left(R^{\prime}\right)^{+}$for all $a^{\prime}, b^{\prime} \in R^{\prime}$. It is clear that, for each $x^{\prime} \in R^{\prime}, x^{\prime} \geq 0$ if and only if $x^{\prime}(\overline{0, e}) \geq 0$.

We show below that $R^{\prime}$ is a lattice-ordered ring under the partial order defined above. In fact, if $a \in S$, then

$$
(\overline{n, a}) \vee 0= \begin{cases}(\overline{n, a \vee 0}) & \text { if } a \vee 0 \in S, \\ (\overline{0, a \vee 0}) & \text { if } a \vee 0 \in T \oplus T^{\perp} .\end{cases}
$$

If $a \in T \oplus T^{\perp}$, then since $T$ is a totally ordered ring and $n e+a_{T} e \in T$, 
$n e+a_{T} e \geq 0$ or $n e+a_{T} e \leq 0$. Thus

$$
(\overline{n, a}) \vee 0= \begin{cases}\left(\overline{n, a_{T}+a_{T \perp} \vee 0}\right) & \text { if } n e+a_{T} e \geq 0, \\ \left(\overline{0, a_{T^{\perp}} \vee 0}\right) & \text { if } n e+a_{T} e \leq 0 .\end{cases}
$$

We show the above results according to the following four cases.

(a) $a \in S$ and $a \vee 0 \in S$. By Lemma 2(a) we have $(\overline{n, a \vee 0}) \geq(\overline{n, a}), 0$. If $b^{\prime}=(\overline{m, b}) \in R^{\prime}$ and $b^{\prime} \geq(\overline{n, a}), 0$, then $m e+b e \geq 0$ and $(m-n) e+(b-a) e \geq$ 0 . If $b \in S$, then $b>0$ by Lemma $2(\mathrm{~b})$, and hence $(m-n) e+b e \geq 0$ by Lemma 2(a). Thus $(m-n) e+b e \geq a e \vee 0=(a \vee 0) e$; that is, $b^{\prime} \geq(\overline{n, a \vee 0})$. If $b \in T \oplus T^{\perp}$, then $b_{T^{\perp}} \geq 0$ by Lemma 4. Since $(b-a) \in S, b-a>0$ by Lemma $2(\mathrm{~b})$, and hence $b_{T^{\perp}}>a$, so $b_{T^{\perp}}>a \vee 0$. Since $b_{T^{\perp}}>a \vee 0, b_{T^{\perp}}$ is in $S$, a contradiction.

(b) $a \in S$ and $a \vee 0 \in T \oplus T^{\perp}$. Since $a=(a \vee 0)-(-a \vee 0)$, we have $(-a \vee 0) \in S$. It follows from (a) that $(\overline{-n,-a}) \vee 0=(\overline{-n,-a \vee 0})$, so $(\overline{n, a}) \vee 0=(\overline{0, a \vee 0})$.

(c) $a \in T \oplus T^{\perp}$ and $n e+a_{T} e \geq 0$. It is evident that $\overline{\left(n, a_{T}+\left(a_{T^{\perp}} \vee 0\right)\right)} \geq$ $(\overline{n, a}), 0$. Let $b^{\prime}=(\overline{m, b}) \in R^{\prime}$ and $b^{\prime} \geq(\overline{n, a}), 0$. Then $m e+b e \geq 0$ and $(m-n) e+(b-a) e \geq 0$. If $b \in S$, then $b>0$, and hence $0<b-a_{T} \in S$. Since $(m-n) e+\left(b-a_{T}\right) e \geq 0$ by Lemma 2 , we have $(m-n) e+\left(b-a_{T}\right) e \geq$ $a_{T^{\perp}} e \vee 0=\left(a_{T^{\perp}} \vee 0\right) e$; that is, $b^{\prime} \geq\left(\overline{n, a_{T}+\left(a_{T^{\perp}} \vee 0\right)}\right)$. If $b \in T \oplus T^{\perp}$, then $b_{T^{\perp}} \geq a_{T^{\perp}} \vee 0$ and $(m-n) e+\left(b_{T}-a_{T}\right) e \geq 0$ by Lemma 4. Thus $(m-n) e+\left(b-a_{T}-a_{T \perp} \vee 0\right) e \geq 0$. Again we have $b^{\prime} \geq\left(\overline{n, a_{T}+\left(a_{T \perp} \vee 0\right)}\right)$.

(d) $a \in T \oplus T^{\perp}$ and $n e+a_{T} e \leq 0$. It is clear that $\left(\overline{0, a_{T^{\perp}} \vee 0}\right) \geq(\overline{n, a}), 0$. If $b^{\prime}=(\overline{m, b}) \in R^{\prime}$ and $b^{\prime} \geq(\overline{n, a}), 0$, then $(m e+b e) \geq 0$ and $(m-n) e+$ $(b-a) e \geq 0$. If $b \in S$, then since $b>0$ and $b>a, 0<\left(b-a_{T^{\perp}} \vee 0\right) \in S$, and hence $m e+\left(b-a_{T^{\perp}} \vee 0\right) e \geq 0$ by Lemma $2(\mathrm{a})$; that is, $b^{\prime} \geq\left(\overline{0, a_{T^{\perp}} \vee 0}\right)$. If $b \in T \oplus T^{\perp}$, then $b_{T^{\perp}} \geq a_{T^{\perp}} \vee 0$ and $m e+b_{T} e \geq 0$ by Lemma 4 . Hence $m e+\left(b-a_{T \perp} \vee 0\right) e \geq 0$. Thus $b^{\prime} \geq\left(\overline{0, a_{T \perp} \vee 0}\right)$.

So far we have shown that $R^{\prime}$ is an l-ring.

Finally, we show that $R^{\prime}$ is squares positive.

Let $a^{\prime}=(\overline{n, a}) \in R^{\prime}, a \in R$. Then $\left(a^{\prime}\right)^{2}=\left(\overline{n^{2}, 2 n a+a^{2}}\right)$. If $a \in S$, by Lemma 5 we have $2 n a+a^{2} \geq 0$, and hence $\left(a^{\prime}\right)^{2} \geq 0$. If $a \in T \oplus T^{\perp}$ and $a=a_{T}+a_{T^{\perp}}$, then $2 n a+a^{2}=2 n a_{T}+2 n a_{T^{\perp}}+a_{T}^{2}+a_{T} a_{T^{\perp}}+a_{T^{\perp}} a_{T}+\left(a_{T^{\perp}}\right)^{2}$, and hence $\left(a^{\prime}\right)^{2}=\left(\overline{n, a_{T}}\right)^{2}+\left(\overline{0,2 n a_{T^{\perp}}+a_{T} a_{T^{\perp}}+a_{T^{\perp}} a_{T}+\left(a_{T^{\perp}}\right)^{2}}\right)$. Since $T$ is a totally ordered ring, $\left(\overline{n, a_{T}}\right)^{2} \geq 0$, and, from Lemma 5 and $a_{T^{\perp}} \in T^{\perp}$, $2 n a_{T^{\perp}}+a_{T} a_{T^{\perp}}+a_{T^{\perp}} a_{T}+\left(a_{T^{\perp}}\right)^{2} \geq 0$, so $\left(a^{\prime}\right)^{2} \geq 0$; that is, $R^{\prime}$ is squares positive.

Let $\psi$ be the map given by $a \rightarrow(\overline{0, a})$ for every $a \in R$. It is evident that $\psi$ is a ring monomorphism from $R$ into $R^{\prime}$ and $\psi(a \vee 0)=(\overline{0, a \vee 0})=$ $(\overline{0, a}) \vee 0=\psi(a) \vee 0$ for each $a \in R$; that is, $\psi$ is an $l$-ring monomorphism.

The proof of Theorem 1 is now complete.

Corollary. An l-semiprime l-ring with squares positive and an $f$-superunit can be embedded in a unital l-semiprime l-ring with squares positive.

Proof. Since an $l$-semiprime $l$-ring with squares positive and an $f$-superunit is a subdirect product of its $l$-prime homomorphic images which are squares positive and contain $f$-superunits, the proof is completed by Theorem 1 . 
Lemma 6. Let $R$ be an l-prime l-ring with squares positive and an $f$-superunit, and let $R^{\prime}$ be as in Theorem 1. If $e$ is an $f$-superunit of $R$, then $(\overline{0, e})$ is an $f$-superunit of $R^{\prime}$.

Proof. Since $e$ is an $f$-superunit of $R,(\overline{1,0}) \leq(\overline{0, e})$, and hence $(\overline{0, e})$ is a superunit of $R^{\prime}$. We show $(\overline{0, e})$ is an $f$-element of $R^{\prime}$. Suppose that $(\overline{n, x}) \wedge(\overline{m, y})=0$. First we show that $(n e+x e) \wedge(m e+y e)=0$ in $R$. Note that $x$ and $y$ are not both in $S$; otherwise, we have $(\overline{1,0}) \leq(\overline{n, x})$, $(\overline{m, y})$ by Lemma 2. If $x \in S$ and $y \in T \oplus T^{\perp}$, then $\left(\overline{m, y_{T}}\right)=0$ and $(\overline{n, x}) \wedge\left(\overline{0, y_{T^{\perp}}}\right)=0$, so $m e+y_{T} e=0$ and $x \wedge y_{T^{\perp}}=0$ since $0 \leq(\overline{0, x}) \leq$ $2(\overline{n, x})$. Thus $(n e+x e) \wedge(m e+y e)=(n e+x e) \wedge y_{T^{\perp}} e=0$. If $x, y \in$ $T \oplus T^{\perp}$, then $\left(\overline{n, x_{T}}\right)=0$ or $\left(\overline{m, y_{T}}\right)=0$ and $\left(\overline{0, x_{T^{\perp}}}\right) \wedge\left(\overline{0, y_{T^{\perp}}}\right)=0$. Thus $(n e+x e) \wedge(m e+y e) \leq\left[\left(n e+x_{T} e\right) \wedge\left(m e+y_{T} e\right)\right]+\left(x_{T^{\perp}} e \wedge y_{T^{\perp}} e\right)=0$, and we get $(n e+x e) \wedge(m e+y e)=0$. Similarly, we also have $(n e+e x) \wedge(m e+e y)=0$. These yield $(\overline{n, x})(\overline{0, e}) \wedge(\overline{m, y})(\overline{0, e})=(\overline{0, n e+x e}) \wedge(\overline{0, m e+y e})=0$ and $(\overline{0, e})(\overline{n, x}) \wedge(\overline{0, e})(\overline{m, y})=(\overline{0, n e+e x}) \wedge(\overline{0, m e+e y})=0$. So $(\overline{0, e})$ is an $f$-element of $R^{\prime}$ since $(\overline{1,0}) \leq(\overline{0, e})$.

Let $R$ be an $l$-prime $l$-ring with squares positive and an $f$-superunit, and let $R^{\prime}$ be as in Theorem 1. It is easy to verify $T\left(R^{\prime}\right)=\{(\overline{n, a}): a \in T\}$ by Lemma 6. Thus $R^{\prime}=S\left(R^{\prime}\right) \cup\left[T\left(R^{\prime}\right)+T\left(R^{\prime}\right)^{\perp}\right]$ by Lemma 1 , where $S\left(R^{\prime}\right)=$ $\{(\overline{n, a}): a \in S\}$ and $T\left(R^{\prime}\right)^{\perp}=\left\{(\overline{0, a}): a \in T^{\perp}\right\}$.

Theorem 2. Let $R$ be an l-prime l-ring with squares positive and an $f$-superunit, and let $R^{\prime}$ be as in Theorem 1 . If $R$ can be embedded as an l-subring in a unital l-ring $B$ and some $f$-superunit of $R$ is an $f$-superunit of $B$, then there are an l-subring $R_{1}$ of $B$ containing $R$ and an l-isomorphism $\psi$ from l-ring $R^{\prime}$ to l-ring $R_{1}$, which satisfies $\psi(a)=a$ for each $a \in R$.

Proof. Let $1_{B}$ be the identity element of $B$ and $R_{1}$ the subring of $B$ generated by $1_{B}$ and $R$. Let $\psi$ be the map from $R^{\prime}$ into $R_{1}$ given by $(\overline{n, a}) \rightarrow n 1_{B}+a$, and let $e$ be an $f$-superunit of $R$ which is also an $f$-superunit of $B$. If $(\overline{n, a})=(\overline{m, b})$, then $(n-m) e+(a-b) e=0$, and hence $\left[(n-m) 1_{B}+(a-b)\right] e=$ 0 (in $B$ ). Since $e$ is an $f$-superunit of $B$, we have $(n-m) 1_{B}+(a-b)=0$, and hence $\psi((\overline{n, a}))=\psi((\overline{m, b}))$; that is, $\psi$ is well defined. It is evident that $\psi$ is an isomorphism of rings and $\psi((\overline{0, a}))=a$. If $a \in R$ and $\left(n 1_{B}+a\right) \vee 0=b$ (in $B)$, then $b e=\left(n 1_{B}+a\right) e \vee 0=(n e+a e) \vee 0$ since $e$ is an $f$-element of $B$. Let $(\overline{n, a}) \vee 0=\left(\overline{n_{1}, a_{1}}\right)$ (in $\left.R^{\prime}\right)$. Since $(\overline{0, e})$ is an $f$-element of $R^{\prime}$ by Lemma 6 , we get $(\overline{n, a})(\overline{0, e}) \vee 0=\left(\overline{n_{1}, a_{1}}\right)(\overline{0, e})$ and $(\overline{0, n e+a e}) \vee 0=$ $\left(\overline{0, n_{1} e+a_{1} e}\right)$, and hence $(n e+a e) \vee 0=n_{1} e+a_{1} e$. Since $R$ is an $l$-subring of $B, b e=n_{1} e+a_{1} e=\left(n_{1} 1_{B}+a_{1}\right) e$, and hence $b=n_{1} 1_{B}+a_{1} \in R_{1}$ since $e$ is an $f$-superunit of $B$; that is, $R_{1}$ is an $l$-subring of $B$. Finally, it is easy to verify that $\psi$ is also an $l$-isomorphism from $R^{\prime}$ to $R_{1}$.

Let $R$ be an $l$-prime $l$-ring with squares positive and an $f$-superunit. Theorem 2 shows that the $R^{\prime}$ in Theorem 1 is the unique smallest unital $l$-ring which contains $R$ and has an $f$-superunit that is an $f$-superunit of $R$. In general, no such uniqueness holds [1, p. 331].

Now we determine the conditions which ensure that an $l$-prime $l$-ring with squares positive which has nonzero $f$-elements can be embedded as a convex $l$-subring in a unital $l$-prime $l$-ring with squares positive. If $R$ is an $l$-prime 
$l$-ring with squares positive which has nonzero $f$-elements, then $R$ is a domain [5, Theorem 1], and hence $T$ is a totally ordered ring. Thus $R=S \cup\left(T \oplus T^{\perp}\right)$ by Lemma 1. In [6, Remark, p. 367] Steinberg defined an l-ring $R$ to be supertessimal if $n|x| \leq\left|x^{2}\right|$ for each $n \in Z^{+}$and $x \in R$.

Theorem 3. Let $R$ be an l-prime l-ring with squares positive which has nonzero $f$-elements, and suppose $R$ has no identity element. $R$ can be embedded as a convex $l$-subring in a unital l-prime l-ring with squares positive if and only if $R$ is supertessimal; or $S=\varnothing, T$ is infinitesimal, and $n|a| \leq a^{2}$ for each $n \in Z^{+}$ and $a \in T^{\perp}$.

Proof. Let $B$ be a unital $l$-prime $l$-ring with squares positive and $R$ a convex $l$-subring of $B$. Since $R$ is a convex $l$-subring of $B, T^{\perp} \subseteq T(B)^{\perp}$, and, also, since $R$ does not have an identity element, $R \subseteq T(B)+T(B)^{\perp}$ by Lemma 1 . If $S \neq \varnothing$, then, for any $a \in S$, we have $|a| \in T(B)^{\perp}$. This yields $S \subseteq T(B)^{\perp}$, and hence $T \subseteq T(B)^{\perp}$, so $R \subseteq T(B)^{\perp}$ by Lemma 1 . From Lemma 5 we get $R$ to be supertessimal. If $S=\varnothing$, but $T$ is not infinitesimal, then there is $d \in T$ which satisfies $0<d<d^{2}$. Let $d=d_{1}+d_{2}$, where $0 \leq d_{1} \in T(B)$ and $0 \leq d_{2} \in T(B)^{\perp}$. Since $R$ is a convex $l$-subring of $B, d_{1}, d_{2} \in R$, and hence $d_{1}, d_{2} \in T$. But $T$ is a totally ordered ring and $d_{1} \wedge d_{2}=0$, so $d_{1}=0$ or $d_{2}=0$. If $d_{2}=0$, then $d \in T(B)$, and hence $d>1_{B}$ (identity element of $B$ ) since $d$ is comparable with $1_{B}$ and $d<d^{2}$. This contradicts the fact that $R$ has no identity element, so $d_{1}=0$ and $d \in T(B)^{\perp}$. This yields $T \subseteq T(B)^{\perp}$, and hence $R \subseteq T(B)^{\perp}$. Thus, again, we get $R$ to be supertessimal by Lemma 5.

Conversely, first suppose $R$ is supertessimal. Let $Z \oplus R$ be as an $l$-group with multiplication defined by $(n, a)(m, b)=(n m, n b+m a+a b)$. Then $Z \oplus R$ is a unital $l$-ring with squares positive [6, p. 367]. If $(n, a)(m, b)=0$, then $n m=0$ and $n b+m a+a b=0$. We may assume $n=0$. If $a \neq 0$, then, since $R$ is domain and $m a+a b=0$, we have $m b+b^{2}=0$, and hence $(|m|+1)|b| \leq b^{2}=-m b=|m b|=|m||b|$ since $R$ is supertessimal. This yields $b=m=0$, and hence $(m, b)=0$; that is, $Z \oplus R$ is a domain. Let $\psi$ be the map given by $a \rightarrow(0, a)$. It is evident that $\psi$ is an $l$-monomorphism and $\psi(R)$ is a convex $l$-subring of $Z \oplus R$.

Finally, let $R$ satisfy the following conditions: $S=\varnothing, T$ is infinitesimal, and $n|a| \leq a^{2}$ for each $a \in T^{\perp}$ and $n \in Z^{+}$. From Lemma 1 we have $R=T \oplus T^{\perp}$.

Let $T^{\prime}$ be the ring obtained by freely adjoining the $Z$ to $T$ with partial order defined by $(n, a) \geq 0$ if and only if $n>0$ or $n=0$ and $a \geq 0$. Then $T^{\prime}$ is a totally ordered domain ring with identity $(1,0)[7,1.4]$.

Let $R^{\prime \prime}=T^{\prime} \oplus T^{\perp}$ as an $l$-group. If $a^{\prime \prime}=\left(\left(n, a_{1}\right), a_{2}\right)$ and $b^{\prime \prime}=\left(\left(m, b_{1}\right)\right.$, $\left.b_{2}\right) \in R^{\prime \prime}$, where $a_{1}, b_{1} \in T$ and $a_{2}, b_{2} \in T^{\perp}$, we define the multiplication in $R^{\prime \prime}$ as

$a^{\prime \prime} b^{\prime \prime}=\left(\left(n, a_{1}\right)\left(m, b_{1}\right)+\left(0,\left(a_{2} b_{2}\right)_{T}\right), n b_{2}+a_{1} b_{2}+m a_{2}+a_{2} b_{1}+\left(a_{2} b_{2}\right)_{T^{\perp}}\right)$.

It is easy to verify $R^{\prime \prime}$ to be a ring with identity $((1,0), 0)$. Since $T$ has mode 0 and $R=T \oplus T^{\perp}, R$ has mode 0 , so $R^{\prime \prime}$ is a domain. Also, the product of positive elements of $R^{\prime \prime}$ is clearly positive by the definition of the multiplication in $R^{\prime \prime}$; that is, $R^{\prime \prime}$ is an $l$-ring.

Let $a^{\prime \prime}=\left(\left(n, a_{1}\right), a_{2}\right) \in R^{\prime \prime}$, where $a_{1} \in T$ and $a_{2} \in T^{\perp}$. Then $\left(a^{\prime \prime}\right)^{2}=$ $\left(\left(n, a_{1}\right)^{2}+\left(0,\left(a_{2}^{2}\right)_{T}\right), 2 n a_{2}+a_{1} a_{2}+a_{2} a_{1}+\left(a_{2}^{2}\right)_{T^{\perp}}\right)$. From the hypothesis and 
Lemma 5, we get $2 n a_{2}+a_{1} a_{2}+a_{2} a_{1}+a_{2}^{2} \geq 0$, and hence $2 n a_{2}+a_{1} a_{2}+a_{2} a_{1}+$ $\left(a_{2}^{2}\right)_{T^{\perp}} \geq 0,\left(a_{2}^{2}\right)_{T} \geq 0$. Thus $\left(a^{\prime \prime}\right)^{2} \geq 0$.

So far we have shown that $R^{\prime \prime}$ is a unital $l$-prime $l$-ring with squares positive. Let $\psi$ be the map from $R$ into $R^{\prime \prime}$ given by $\psi(a)=\left(\left(0, a_{T}\right), a_{T^{\perp}}\right)$ for each $a \in R$. It is clear that $\psi$ is an $l$-monomorphism. If $\left(\left(n, a_{1}\right), a_{2}\right)$ and $\left(\left(0, b_{1}\right), b_{2}\right) \in R^{\prime \prime}$ satisfy $0 \leq\left(\left(n, a_{1}\right), a_{2}\right) \leq\left(\left(0, b_{1}\right), b_{2}\right)$, then $n=0$ by the definition of the partial order of $R^{\prime \prime}$, and hence $\psi(R)$ is a convex $l$-subring of $R^{\prime \prime}$.

The proof of Theorem 3 is now completed.

\section{ACKNOWLEDGMENT}

The author thanks the referee for simplifying the proofs of Theorems 1 and 3.

\section{REFERENCES}

1. S. A. Steinberg, On the unitability of a class of partially ordered rings that have squares positive, J. Algebra 100 (1986), 325-343.

2. __ Unital l-prime lattice-ordered rings with polynomial constraints are domains, Trans. Amer. Math. Soc. 276 (1983), 145-164.

3. D. G. Johnson, A structure theory for a class of lattice-ordered rings, Acta. Math. 104 (1960), 163-215.

4. P. Conrad, Some structure theorems for lattice-ordered groups, Trans. Amer. Math. Soc. 99 (1961), 212-240.

5. Ma Jingjing, On lattice-ordered rings with polynomial constraints, J. Math. Res. Exposition 11 (1991), 325-330. (Chinese)

6. S. A. Steinberg, On lattice-ordered rings in which the square of every element is positive, J. Austral. Math. Soc. 22 (1976), 362-370.

7. M. Henriksen and J. Isbell, Lattice-ordered rings and function rings, Pacific J. Math. 12 (1962), 533-565.

Number 911 Door 9 Building 11, 23 Xin Wai Street, Beijing 100088, People's Republic OF CHINA

Current address: Department of Mathematics, The University of Toledo, Toledo, Ohio 43606

E-mail address: fac33300uof t01. UTOLEDO.edu 